Pathologe 2010 · 31:471-476

DOI 10.1007/s00292-010-1364-4

(c) Springer-Verlag 2010

\author{
G. Jundt ${ }^{1,2} \cdot$ D. Baumhoer ${ }^{2}$ \\ ${ }^{1}$ Knochentumor-Referenzzentrum am Institut für Pathologie, \\ Universitätsspital Basel, Schweiz \\ ${ }^{2}$ Institut für Pathologie, Universitätsspital Basel, Schweiz
}

\title{
Familiäre
}

\section{Tumorerkrankungen im Knochen}

sarkome) und eine ungewöhnliche Lokalisation auf $[15,17]$.

Chondrosarkome können im Rahmen der so genannten Exostosenkrankheit/ multiple hereditäre Exostosen [6] oder der Enchondromatose/M. Ollier und Maffucci-Syndrom [22] auftreten (• Tab. 1). Obwohl die beiden letztgenannten Entitäten überwiegend sporadisch vorkommen, werden sie in diese Darstellung mit aufgenommen, da familiäre Fälle bekannt sind $[27]$.

\section{Retinoblastomsyndrom}

Das Retinoblastomsyndrom geht mit der Entwicklung von intraokularen malignen Tumoren einher, die sich von primitiven Netzhautzellen ableiten, in 50\% der Fälle familiär auftreten und eine autosomal- dominante Vererbung zeigen. Die meisten dieser Patienten entwickeln bilaterale Augentumoren. Nichthereditäre Retinoblastome hingegen sind zu etwa zwei Dritteln unilateral.

Verantwortlich für die Tumorenstehung sind Mutationen des Retinoblastomgens $(R B 1)$, das auf dem Chromosom 13q14.1 lokalisiert ist. Bei der hereditären Form liegen bereits Keimbahnmutationen eines Allels vor, zu der im weiteren Verlauf eine zusätzliche somatische Mutation des anderen Allels hinzukommt. Beide führen zu einer kompletten Inaktivierung des Retinoblastomgens, das eine zentrale Rolle in der Regulation des Zellzyklus spielt [4]. Bei sporadisch auftretenden Retinoblastomen sind beide Mutationen des $R B_{1}$ Gens erworben. Patienten mit $R B 1$-Mutationen entwickeln häufig weitere maligne

Tab. 1 Familiäre Knochentumoren

\begin{tabular}{|c|c|c|c|c|c|}
\hline $\begin{array}{l}\text { OMIM- } \\
\text { Nummer }\end{array}$ & Name & Gen & Chromosom & $\begin{array}{l}\text { Vererbungs- } \\
\text { modus }\end{array}$ & Tumortyp \\
\hline 180200 & Retinoblastomsyndrom & $R B 1$ & $13 q 14.1-q 14.2$ & $A D$ & Osteosarkome, Weichteiltumoren \\
\hline 151623 & $\begin{array}{l}\text { Li-Fraumeni-Syndrom } \\
\text { „Li-Fraumeni-like-Syndrom“ }\end{array}$ & p53 & $17 p 13.1$ & $A D$ & Osteosarkome, verschiedene andere maligne Tumoren \\
\hline 268400 & Rothmund-Thomson-Syndrom & RECQL4 & $8 q 24.3$ & AR & Osteosarkome, andere Tumoren \\
\hline 277700 & Werner-Syndrom & WRN & $8 p 12-p 11.2$ & AR & Osteosarkome, andere Knochen- und Weichteilsarkome \\
\hline 210900 & Bloom-Syndrom & $B L M$ & $15 q 26.1$ & AR & $\begin{array}{l}\text { Osteosarkome, außerdem NHL, Leukämien, verschie- } \\
\text { dene Karzinome }\end{array}$ \\
\hline 133700 & Multiple hereditäre Exostosen, Typ I & EXT1 & $8 q 24.11-q 24.13$ & $A D$ & Osteochondrome, sekundäre Chondrosarkome \\
\hline 133701 & Multiple hereditäre Exostosen, Typ II & EXT2 & $11 p 12-p 11$ & $A D$ & Osteochondrome, sekundäre Chondrosarkome \\
\hline 166000 & $\begin{array}{l}\text { Enchondromatose, } \\
\text { Maffucci-Syndrom }\end{array}$ & PTHR1(?) & - & $\begin{array}{l}\text { Wenige fami- } \\
\text { liäre Fälle }\end{array}$ & $\begin{array}{l}\text { Enchondrome, (Maffucci: zusätzlich vaskuläre Läsio- } \\
\text { nen), sekundäre Chondrosarkome, andere Tumoren }\end{array}$ \\
\hline
\end{tabular}



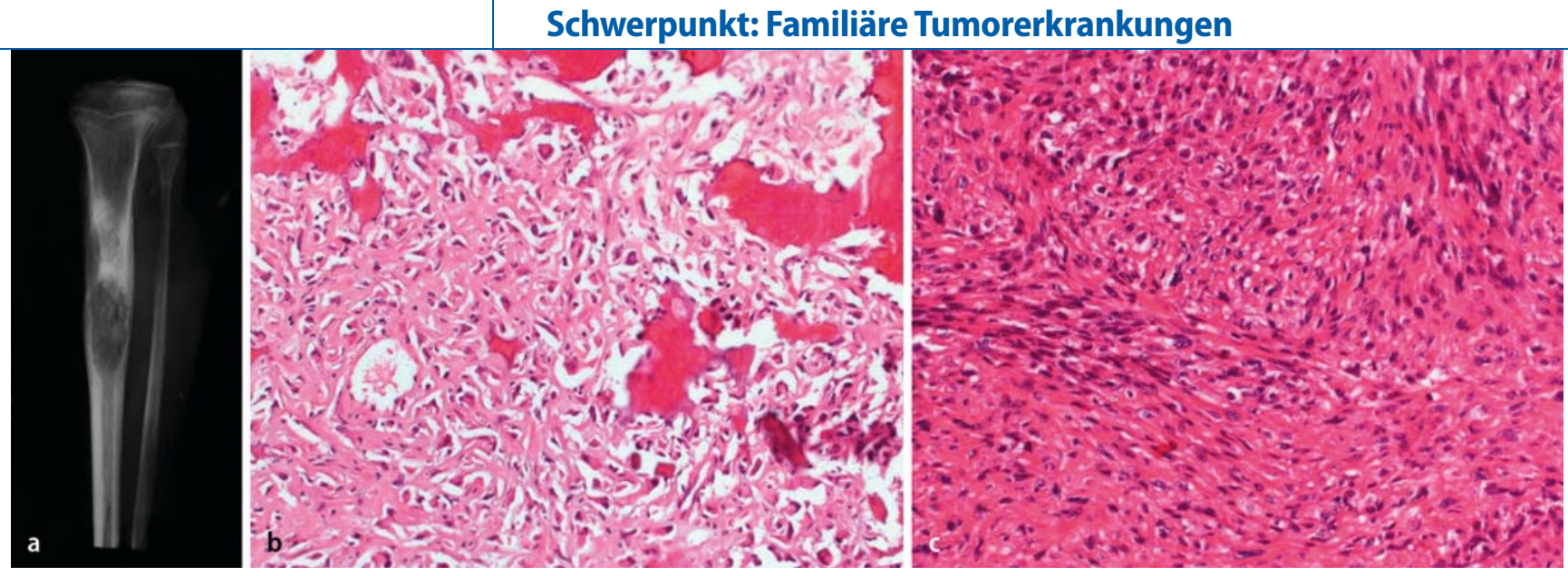

Abb. 1 \ Osteosarkom bei Rothmund-Thomson-Syndrom. a Präparatradiogramm. 13-jähriger Patient mit diaphysärem Osteosarkom der Tibia. Im oberen Anteil sind vorwiegend sklerotische, im unteren Anteil in Schaftmitte vorwiegend osteolytische Areale erkennbar. b Irreguläre, breite mineralisierte Matrixanteile (obere Präparathälfte) sowie gitterförmiges nichtmineralisiertes Tumorosteoid sichern die Diagnose eines Osteosarkoms. c Ausgedehnte Areale des Osteosarkoms zeigen eine fibroblastische Differenzierung ohne Osteoidbildung. Diese Bezirke stammen aus der in Schaftmitte gelegenen Osteolyse

Tumoren, wobei sich 50 Jahre nach der Erstdiagnose eine kumulative Zweittumorinzidenz von 51\% ergibt [7]. Am häufigsten werden Osteosarkome und Weichteilsarkome, besonders Leiomyosarkome, gefunden, gefolgt von Karzinomen, Hirntumoren, Melanomen, Leukämien und anderen Tumoren (Literatur bei [21]). Diese Sekundärtumoren können in 5 Gruppen unterteilt werden [7]:

1. Tumoren, die sich im Bestrahlungsfeld entwickeln,

2. Tumoren, die außerhalb des Bestrahlungsfeldes entstehen,

3. Tumoren bei Patienten, die keine Radiotherapie erhalten haben,

4. Tumoren, bei denen nicht entschieden werden kann, ob es sich um Primärtumoren oder Metastasen handelt,

5. Tumoren bei Patienten, die aus einer Retinoblastomfamilie stammen, selbst aber kein Retinoblastom aufweisen.

$R B 1$-Alterationen, die zu einer verminderten Expression des Genprodukts führen, prädisponieren nach tierexperimentellen Befunden offenbar außerdem zur Entwicklung strahleninduzierter Osteosarkome, unabhängig vom Vorliegen eines Retinoblastoms ([33]; M. Rosemann, persönliche Mitteilung). So konnten Chauveinc et al. bei Retinoblastompatienten ein um etwa 12 Monate vorverlegtes Auftreten von Osteosarkomen im Bestrahlungsfeld im Gegensatz zum Auftreten von Os- teosarkomen außerhalb des Bestrahlungsfeldes oder bei nichtbestrahlten Retinoblastompatienten beobachten [8].

Histologie, Lokalisation und Therapie sekundärer, Retinoblastom-assoziierter Osteosarkome sind die gleichen wie bei sporadischen konventionellen, primär auftretenden Osteosarkomen [1, 24].

\section{RecQ-Helicasen-assoziierte Syndrome}

Das Rothmund-Thomson-Syndrom, das Bloom-Syndrom und das Werner-Syndrom werden durch Genmutationen hervorgerufen, die die Familie der so genannten RecQ-Helicasen betreffen. Dabei handelt es sich um eine hoch konservierte Familie von Proteinen, die zur genetischen Stabilität beitragen und somit einer neoplastischen Transformation entgegenwirken. Benannt wurden sie nach dem Produkt des recQ-Gens in Escherichia coli. Menschliche Zellen enthalten 5 dieser RecQ-Helicasen, wobei mindestens 3 von ihnen als Tumorsuppressor wirken [9]. Zu ihnen gehören das RECQL4-, das auch als RECQ4 bezeichnet wird, das BLM- und das WRN-Gen. Keimbahnmutationen führen zu den genannten Syndromen, die mit einer erhöhten Krebsentwicklung einhergehen [14].

\section{Rothmund-Thomson-Syndrom}

Das Rothmund-Thomson-Syndrom (RTS) ist eine seltene autosomal-rezes- sive Erkrankung, die Männer doppelt so häufig wie Frauen betrifft. Es wird bereits im ersten Lebensjahr beobachtet und ist charakterisiert durch eine sonnenabhängige Poikilodermie, eine kurze Statur, ossäre Auffälligkeiten und andere Veränderungen. In etwa $30 \%$ der Fälle entwickeln sich konventionelle Osteosarkome, die charakteristischerweise bereits sehr früh (Durchschnittsalter 11 Jahre) und an ungewöhnlichen Orten (z. B. diaphysär - Abb. 1) auftreten $[20,35]$. Auch multifokale Osteosarkome sind bei dieser Erkrankung beobachtet worden.

Ursächlich scheint eine Mutation des RECQL4-Gens auf Chromosom 8q24.3 zu sein, das für eine DNA-Helicase kodiert, die doppelsträngige DNA in einzelsträngige DNA aufspaltet. Wang et al. konnten zeigen, dass Mutationen, die mit einem Bruch einhergehen, zu einem Funktionsverlust des Gens führen und in RTS-Patienten mit einem hohen Risiko einer Osteosarkomentwicklung vergesellschaftet sind [34].

\section{Bloom-Syndrom}

Das Bloom-Syndrom, das 1954 von Bloom erstmals als kongenitales, dem Lupus erythematodes ähnliches teleangiektatisches Erythem beschrieben wurde [3], gehört ebenfalls zu den RecQ-Helicase-assoziierten Tumorsydromen. Es geht mit Kleinwuchs einher und ist mit einem erhöhten Risiko behaftet, maligne Tumoren, darunter Osteosarkome, zu entwickeln. Betrof- 
fen ist ein Gen einer DNA-Helicase, BLM, das auf Chromosom 15q26.1 lokalisiert ist. Die Erkrankung ist extrem selten, bisher wurden etwa 230 Fälle publiziert [9].

\section{Werner-Syndrom}

Anfang des letzten Jahrhunderts (1904) beschrieb Werner in seiner Dissertation („Über Katarakt in Verbindung mit Sklerodermie") eine seltene autosomalrezessive Erkrankung, die mit einer vorzeitigen Alterung, einer Entwicklung von Katarakten, subkutanen Kalzifikationen, frühzeitiger Arteriosklerose, einem Diabetes mellitus, einer vorzeitigen Gesichtsalterung und einem hohen Risiko einer Tumorentwicklung (besonders Weichteilsarkome, Osteosarkome, Melanome und Schilddrüsenkarzinome; oft Mehrfachtumoren) einhergeht, wobei das Verhältnis von Karzinomen zu Sarkomen 1:1, und nicht wie in der übrigen Bevölkerung 10:1 beträgt $[25,36]$. Die Erkrankung kommt hauptsächlich in Japan vor. Das WRN-Gen konnte auf Chromosom 8p12-p11 identifiziert werden. Das WRNProtein gehört ebenfalls in die Gruppe der RecQ-Proteine, wobei es sowohl als DNAHelicase als auch als Exonuclease aktiv ist. Besonders Alterationen der Helicasefunktionen können zu chromosomaler Instabilität und konsekutiver Krebsentwicklung führen [14]. Bisher sind über 50 verschiedene Mutationen des WRN-Gens beschrieben worden [25].

Patienten mit Werner-Syndrom, die ein Osteosarkom entwickeln, sind in der Regel älter (um die 40 Jahre). Meist sind die distalen Skelettabschnitte vor allem der unteren Extremität (Knöchelregion, Fuß, Patella) betroffen. Alle histologischen Subtypen können vertreten sein [17].

\section{Li-Fraumeni-Syndrom}

Das Li-Fraumeni-Syndrom (LFS) wurde 1988 erstmals als autosomal-dominant vererbte Erkrankung beschrieben, die durch die Entwicklung multipler Primärtumoren bereits im jungen Erwachsenenalter charakterisiert ist [19]. Vornehmlich entwickeln sich Weichteilsarkome, Osteosarkome, prämenopausale Mammakarzinome und Hirntumoren, außerdem Leukämien und Nebennierenrindenkar-

\section{Pathologe 2010 · 31:471-476 DOI 10.1007/s00292-010-1364-4}

(c) Springer-Verlag 2010

\section{G. Jundt $\cdot$ D. Baumhoer \\ Familiäre Tumorerkrankungen im Knochen}

\section{Zusammenfassung}

Familiäre Erkrankungen, die zur Bildung von Knochentumoren führen, sind selten. Sie entwickeln sich im Zusammenhang mit genetischen Alterationen, die den Zellzyklus (Retinoblastomsyndrom/RB1, Li-Fraumeni-Syndrom/p53), wachstumssteuernde Transkriptionskaskaden (Enchondromatose/PTHR1, multiple hereditäre Exostosen/EXT1, EXT2) oder den Erhalt der DNA-Stabilität (Rothmund-Thomson-/RECQL4, Werner-/WRN und Bloom-Syndrom/BLM) betreffen. So entstehen entweder multiple gutartige Tumoren, die sekundär maligne transformieren (Enchondromatosen: Enchondrome; multiple hereditäre Exostosen: Osteochondrome), oder maligne Tumoren, meist Osteosarkome

\section{Hereditary bone tumors}

\section{Abstract}

Familial diseases leading to bone tumor formation are rare. They are mainly caused by genetic alterations of cell cycle constituent genes, such as retinoblastoma syndrome (RB1) and Li-Fraumeni syndrome ( $p 53)$, of genes involved in growth-regulating transcriptional cascades, such as enchondromatosis (PTHR1) and multiple hereditary exostoses (EXT1, EXT2) or of genes maintaining chromosomal stability, such as RothmundThomson (RECQL4), Werner (WRN) and Bloom syndromes (BLM). This leads to multiple benign bone tumors, which may undergo secondary malignant transformation (enchondromatosis: enchondromas, multiple hereditary exostoses: osteochondromas) or bone sarcomas, mainly osteosarcomas, such als Primär- (Li-Fraumeni-, Rothmund-Thomson-, Werner- und Bloom-Syndrom) oder Sekundärmanifestation (Retinoblastomsyndrom). Einige dieser Läsionen sind außerdem mit einem erhöhten allgemeinen Tumorrisiko behaftet. Gegenüber sporadisch auftretenden gleichartigen Tumoren bestehen oft Unterschiede in zeitlicher Manifestation, Lokalisation und Histologie, die für die Erkennung des zugrunde liegenden Syndroms hilfreich sein können.

\section{Schlüsselwörter}

Retinoblastomsyndrom · Li-FraumeniSyndrom · Rothmund-Thomson-Syndrom . Werner-Syndrom · Bloom-Syndrom

as primary (Li-Fraumeni, Rothmund-Thomson, Werner and Bloom syndromes) or secondary manifestations (retinoblastoma syndrome) of the underlying disease. Some of these lesions also carry an increased risk for developing additional malignant diseases. In contrast to sporadically occurring similar tumors, differences in manifestation in time, topography or histology may be present which can aid in the correct recognition of the underlying syndrome.

\section{Keywords}

Retinoblastoma syndrome $\cdot$ Li-Fraumeni syndrome $\cdot$ Rothmund-Thomson syndrome . Werner syndrome $\cdot$ Bloom syndrome 
zinome. Diese Tumoren treten vor dem 45. Lebensjahr auf und betreffen Patienten, deren erst- oder zweitgradige Verwandte ebenfalls eine Krebserkrankung vor dem 45. Lebensjahr oder ein Sarkom $\mathrm{zu}$ irgendeinem Lebensalter entwickelt haben.

Nach der Definition von Li und Fraumeni wird von einem klassischen LFS gesprochen, wenn

- bei einem Patienten ein Sarkom vor dem 45. Lebensjahr auftritt,

- ein Verwandter ersten Grades ebenfalls eine Krebserkrankung vor dem 45. Lebensjahr entwickelt hat und

- ein weiterer Verwandter ersten oder zweiten Grades an einem Krebsleiden vor dem 45. Lebensjahr oder einem Sarkom zu irgendeinem Lebensalter erkrankt war [19].

Davon unterschieden wird das so genannte "Li-Fraumeni-like-Syndrom“ (LFL). Unter diese Definition fallen

- alle Patienten mit einer Krebserkrankung im Kindesalter oder einem Sarkom, Hirntumor oder Nebennierenrindenkarzinom vor dem 45 . Lebensjahr,

- deren erst- oder zweitgradige Verwandte einen typischen LFS-Tumor (Sarkom, Mammakarzinom, Hirntumor, Leukämie, Nebennierenrindenkarzinom) zu irgendeinem Lebensalter entwickelt haben und

- bei denen zusätzlich ein weiterer erstoder zweitgradiger Verwandter in gleicher Linie an einem Krebsleiden vor dem 6o. Lebensjahr erkrankt ist [2].

Diese Kriterien sind mittlerweile durch einzelne Arbeitsgruppen weiter modifiziert und ergänzt worden (Literatur bei $[13,32])$.

In etwa 70\% der Fälle lassen sich Keimbahnmutationen des p53-Gens nachweisen, das auf dem Chromosom 17p13.1 lokalisiert ist $[26,32]$. Ji und Hemminki konnten in einer epidemiologischen Studie aus Schweden außerdem zeigen, dass das Risiko einer Osteosarkomentwicklung bei Patienten vor dem 25. Lebensjahr, deren Mütter an einem Mammakarzinom oder Melanom erkrankt waren, 1,7- bzw. 2,9-fach höher war als zu erwarten [18].
Möglicherweise liegt bei diesen Patienten eine Assoziation mit einer anderen Form des LFS oder LFL vor. Die Osteosarkome unterscheiden sich hinsichtlich Lokalisation und Altersverteilung nicht wesentlich von den bekannten sporadischen Formen. Eine ungewöhnliche Histologie (teleangiektatisch, riesenzellreich, fibroblastisch) scheint jedoch häufiger zu sein und sollte zur Erhebung der Familienanamnese veranlassen [15].

\section{Enchondromatose}

Die Enchondromatose ist durch die Entwicklung zahlreicher Enchondrome gekennzeichnet, die gewöhnlich die Metaphysen und Diaphysen der Röhrenknochen betreffen [22]. Es bestehen zahlreiche Subgruppen, die aufgrund klinischer Besonderheiten unterschieden werden. Falls die Skelettbeteiligung ausgeprägt ist, besonders wenn der Befall vorwiegend einseitig ist (wie von Ollier 1899 primär beschrieben), wird die Bezeichnung $M$. Ollier verwendet.

Die Erkrankung tritt überwiegend sporadisch auf, seltene familiäre Fälle sind jedoch publiziert (Literatur bei [27]). Molekulargenetisch scheint eine Mutation des Parathyreoidhormonrezeptor-1-Gens $(P T H R 1)$ konstitutiv für die Erkrankung zu sein [16]. Andere Untersucher konnten diese Befunde jedoch nicht bestätigen [29]. Neuerdings wurden zusätzliche, bisher nicht bekannte Mutationen des PTHR1-Gens gefunden, die ebenfalls zu einer Beeinträchtigung der PTHR1-Funktion und der von diesem Rezeptor gesteuerten Signalkaskade führen [11].

Beim Maffucci-Syndrom handelt es sich um eine Kombination von Gefäßfehlbildungen (venöse Malformationen oder Spindelzellhämangiome) und Enchondromen, die erstmals von Maffucci 1881 beschrieben worden ist (zitiert nach [10]). Die Gefäßläsionen entwickeln sich an der Haut, im subkutanen Fettgewebe („kavernöse Hämangiome“), aber auch viszeral, und können unilateral oder bilateral, lokalisiert oder multifokal auftreten. Die Knorpeltumoren treten in der Regel einseitig auf.

Sowohl bei der Enchondromatose als auch beim Maffucci-Syndrom sind Femur, Tibia und Ilium häufig befallen, da- neben die Phalangen, Metacarpalia und Metatarsalia. Das kraniofaziale Skelett, die Wirbelsäule, die Hand- und Fußwurzelknochen sind nur selten betroffen.

Klinisch werden die Patienten durch knotige Schwellungen auffällig, die besonders an den Fingern sichtbar sind. Außerdem treten Längenwachstumsstörungen auf, die schon bei kleinen Kindern zu einem Hinken führen können. Schmerzen werden nicht angegeben, außer bei pathologischen Frakturen. Die Erkrankung kann mit der Pubertät zum Stillstand kommen, gelegentlich jedoch auch fortschreiten.

Histologisch findet sich intramedullär, gelegentlich auch subperiostal, knotig aufgebautes, sehr zellreiches (auch in den langen Röhrenknochen!) hyalines Knorpelgewebe, jedoch keine myxoide Umwandlung der Matrix. Die Kerne können vergrößert und irregulär gestaltet sein.

Hauptkomplikation ist die Entwicklung von Chondrosarkomen, wobei offenbar für jedes Enchondrom die gleiche Wahrscheinlichkeit einer malignen Entartung besteht, sodass das Gesamtrisiko wohl eher mit der Anzahl der Enchondrome zusammenhängt. Das Risiko einer Chondrosarkomentwicklung scheint aber beim Maffucci-Syndrom deutlich höher $\mathrm{zu}$ sein [22]. Meist entstehen niedrig maligne Chondrosarkome, die bei adäquater Therapie eine relative gute Prognose bezogen auf den einzelnen Tumor haben, die sich nicht von der der sporadischen Formen unterscheidet und von Tumorgröße, Lokalisation und Malignitätsgrad abhängig ist $[22,30]$. Die Tendenz zur malignen Entartung besteht auch in den kleinen Röhrenknochen ( $\bullet$ Abb. 2), in denen bei sporadischen Formen sehr selten maligne Transformationen beobachtet werden [31].

Bei beiden Erkrankungen sind außerdem Angiosarkome, Hirntumoren und hepatobiliäre Neoplasien beschrieben worden, die häufig für die Lebenserwartung der Patienten bedeutsamer sind ( $\mathrm{Li}$ teratur bei [28]).

\section{Multiple hereditäre Exostosen}

Multiple hereditäre Exostosen repräsentieren eine familiäre Erkrankung (Exostosenkrankheit bzw. familiäre Osteochondro- 

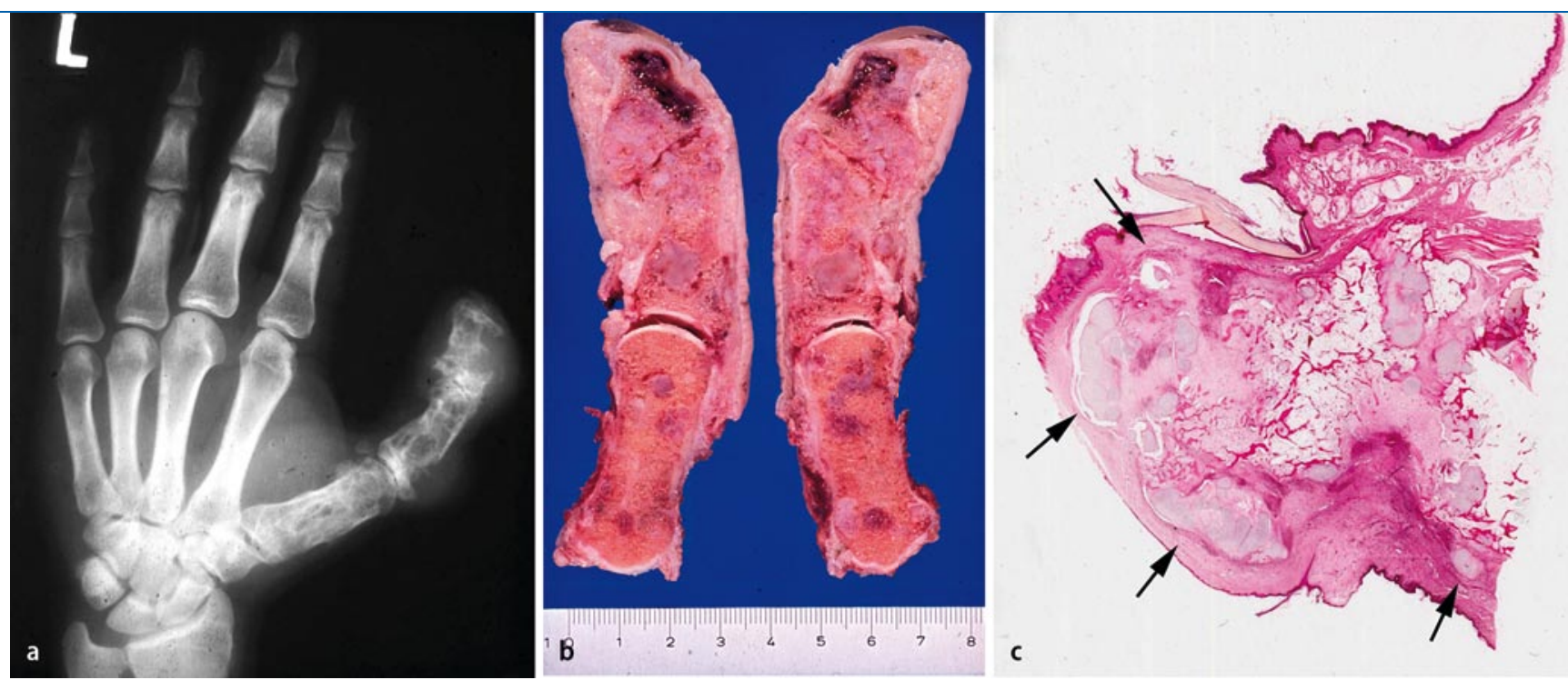

Abb. $2 \Delta$ Enchondromatose mit Übergang in sekundäres Chondrosarkom. 29-jähriger Patient, bei dem vor 13 Jahren erstmals eine Enchondromatose diagnostiziert wurde, die sich klinisch mit Schmerzen und Druckgefühl bemerkbar gemacht hatte. a Wiedervorstellung wegen erneuter Größenzunahme und Schmerzen. b Amputat des Daumens. Die knotige Konfiguration der Knorpeltumoren ist makroskopisch im Metakarpale, der Grund- und Endphalanx gut zu erkennen. Bereits makroskopisch sind Infiltrationen der Weichteile sichtbar (z. B. Grenze Grundphalanx/Endphalanx). c Der Tumor hat die Kortikalis der Endphalanx durchbrochen (Pfeile) und breitet sich ins Weichgewebe aus
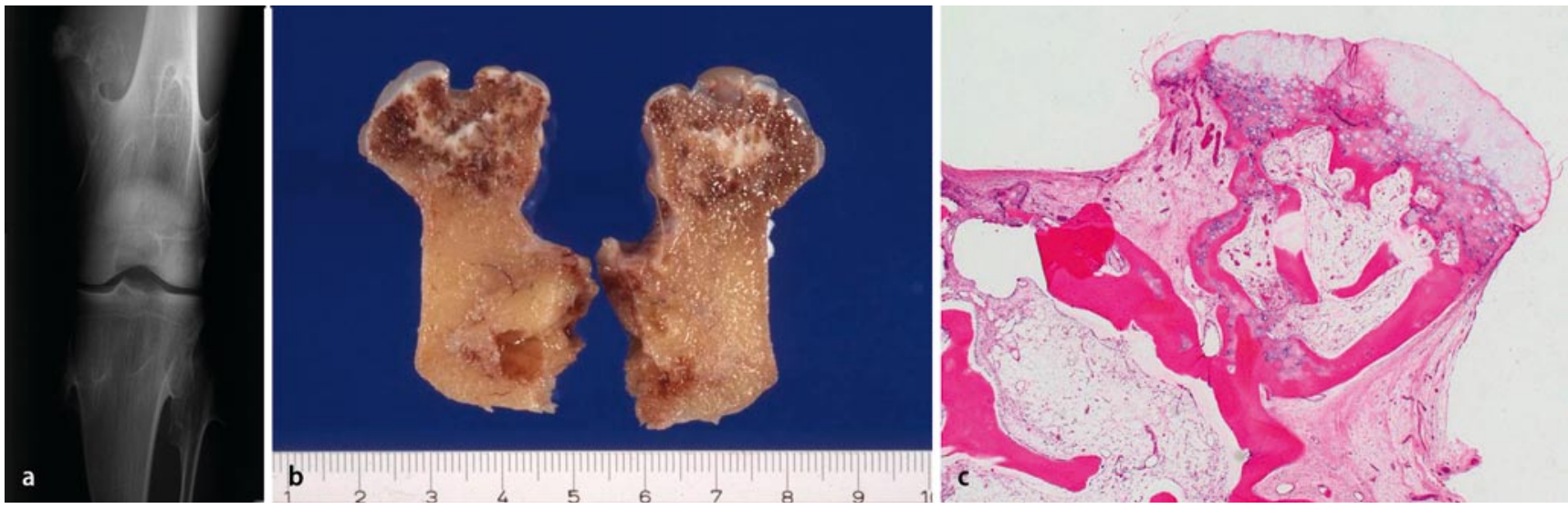

Abb. $3 \Delta$ Multiple hereditäre Exostosen bei einem 20-jährigen Patienten mit bekannter familiärer Exostosenkrankheit. a Wegen störender Irritation Abtragung des am distalen Femur befindlichen medial gelegenen Osteochondroms (oben links). b Auf der Sägeschnittfläche ist der kontinuierliche Übergang des Osteochondromstils in den Markraum gut erkennbar. Die Knorpelkappe ist sehr schmal und offenbar durch Traumatisierungen alteriert. c. Das Osteochondrom zeigt eine schmale, die Wachstumsfugenorganisation imitierende Knorpelkappe, die im linken Anteil offenbar traumatisiert wurde. Hier ist der Knorpel durch eine gefäßreiche, narbenähnliche Fibrosezone ersetzt

matose), die autosomal-dominant vererbt wird und eine niedrigere Penetranz bei Frauen aufweist. Männer sind in einem Verhältnis von 1,5:1 häufiger als Frauen betroffen. Die Inzidenz liegt bei 1:50.000. Etwa 15\% aller Patienten mit Osteochondromen zeigen multiple Tumoren. In der Regel wird die Erkrankung schon im Kleinkindesalter manifest [6].

Die Diagnose wird meist radiologisch gestellt und erfordert den Nachweis von mindestens 2 juxtaepiphysär gelegenen Osteochondromen an den langen Röhren- knochen [5]. Die Knieregion, Hüfte, Knöchel und Schulter sind am häufigsten betroffen, wobei Wachstumsstörungen besonders an den Unterarmen und Beinen beobachtet werden können ( $\boldsymbol{\bullet}$ Abb. 3). $\mathrm{Da}$ die Läsionen häufig im Bereich der metaphysären Wachstumszone lokalisiert sind, kann eine asymmetrische Wachstumsretardierung resultieren. Viele Patienten klagen über Schmerzen.

Meist manifestieren sich die Läsionen als so genannte sessile Osteochondrome, d. h. sie sitzen halbkugelig dem Knochen auf und bilden relativ selten einen schlanken Stiel. Eine maligne Transformation in Richtung Chondrosarkom kann vorkommen und ist sicher bei Patienten mit Osteochondromatose häufiger als bei Patienten mit solitären Osteochondromen, selbst wenn man das statistische Risiko für die einzelne Läsion betrachtet.

Sehr selten treten Osteosarkome oder Fibrosarkome auf, dann jedoch nicht im Bereich der Knorpelkappe, sondern an der Basis bzw. am Übergang zum Markraum. Genaue Zahlen liegen nicht vor. Die An- 
gaben in der Literatur schwanken zwischen 5-25\%, die jedoch in spezialisierten Zentren erhoben wurden. Studien betroffener Familien weisen eindeutig auf einen niedrigeren Prozentsatz (0,5-5\%) mit sarkomatöser Transformation hin. Die Entwicklung peripherer (epiexostotischer) Chondrosarkome wird in der Regel von einer Größenzunahme des Tumors (nach der Pubertät) und einer Zunahme der Schmerzintensität begleitet. Eine Verbreiterung der Knorpelkappe über $1,5 \mathrm{~cm}$ kann radiologisch (T2-gewichtete MRTSequenzen) ein Hinweis sein.

Die Erkrankung ist auf Mutationen im $E X T_{1}$ - und EXT2-Gen zurückzuführen, die auf Chromosom 8q24.1 und Chromosom 11p11-p12 lokalisiert sind. Da durch moderne, sensitivere Methoden die Zahl der Fälle deutlich zurückgegangen ist, bei denen weder eine $E X T_{1}$ - noch eine $E X T_{2}$ Mutation gefunden werden konnte, ist die früher postulierte Existenz eines „EXT3Gens" sehr unwahrscheinlich geworden.

Die von diesen Genen kodierten Glykoproteine spielen eine Rolle bei der Biosynthese der Knorpelmatrix (Heparansulfatproteoglycanbiosynthese). Alteriertes Heparansulfat scheint im Knorpelgewebe die Diffusion von „Indian Hedgehog“ (IHh) zu seinem Rezeptor PTCH zu beeinträchtigen und damit die negative Wachstumsregulation von proliferierenden Chondrozyten durch IHh in der Knorpelkappe eines Osteochondroms aufzuheben [5].

Ein besonderes Risiko für eine Sarkomentwicklung scheint für Patienten mit einer Mutation des EXT1-Gens zu bestehen. Entsprechende genetische Untersuchungen sollten deshalb durchgeführt werden [12].

\section{Fazit für die Praxis}

Auch wenn syndromassoziierte Knochentumoren sehr selten sind, sollte der $\mathrm{Pa}$ thologe bei der Diagnose eines Knochentumors den Verdacht auf das Vorliegen eines hereditären bzw. kongenitalen Knochentumors äußern, wenn - der Patient ein für die Läsion ungewöhnliches Alter aufweist, - der Tumor eine ungewöhnliche Lokalisation besitzt und/oder

_ der Tumor eine ungewöhnliche Histologie zeigt.
Der Kliniker kann dann unter Berücksichtigung der Familienanamnese weitere Untersuchungen und evtl. eine genetische Beratung veranlassen.

\section{Korrespondenzadresse}

\section{Prof. Dr. G. Jundt}

Knochentumor-Referenzzentrum am Institut für Pathologie, Universitätsspital Basel

Schönbeinstr. 40, 4031 Basel

Schweiz

gernot.jundt@unibas.ch

Interessenkonflikt. Der korrespondierende Autor gibt an, dass kein Interessenkonflikt besteht.

\section{Literatur}

1. Bielack SS, Kempf-Bielack B, Heise U et al (1999) Combined modality treatment for osteosarcoma occurring as a second malignant disease. J Clin Oncol 17:1164-1174

2. Birch JM, Hartley AL, Tricker KJ et al (1994) Prevalence and diversity of constitutional mutations in the p53 gene among 21 Li-Fraumeni families. Cancer Res 54:1298-1304

3. Bloom D (1954) Congenital telangiectatic erythema resembling lupus erythematosus in dwarfs; probably a syndrome entity. AMA Am J Dis Child 88:754-758

4. Bornfeld N, Schüler A, Bölöni R et al (2006) Retinoblastom. Ophthalmologe 103:59-78

5. Bovee J (2008) Multiple osteochondromas. Orphanet J Rare Dis 3:3

6. Bovée J, Hogendoorn P (2002) Multiple osteochondromas. In: Fletcher CDM, Unni KK, Mertens F (eds) WHO: Pathology and genetics of tumours of the soft tissue and bone. IARC, Lyon, Sp360-362

7. Cavenee W, Bögler O, Hadjistilianou T et al (2002) Retinoblastoma syndrome. In: Fletcher C, Unni K, Mertens $F$ (eds) Tumours of soft tissue and bone. IARC, Lyon, p 363-364

8. Chauveinc L, Mosseri V, Quintana E et al (2001) Osteosarcoma following retinoblastoma: age at onset and latency period. Ophthalmic Genet 22:7788

9. Chu WK, Hickson ID (2009) RecQ helicases: multifunctional genome caretakers. Nat Rev Cancer 9:644-654

10. Ciranni R (2006) A forgotten Italian pathologist: Angelo Maffucci (1845-1903) and his scientific thought. Virchows Archiv 449:495-497

11. Couvineau A, Wouters V, Bertrand G et al (2008) PTHR1 mutations associated with Ollier disease result in receptor loss of function. Hum Mol Genet 17:2766-2775

12. Francannet $C$, Cohen-Tanugi $A$, Le Merrer $M$ et al (2001) Genotype-phenotype correlation in hereditary multiple exostoses. J Med Genet 38:430-434

13. Gonzalez KD, Noltner KA, Buzin CH et al (2009) Beyond Li Fraumeni syndrome: clinical characteristics of families with p53 germline mutations. J Clin Oncol 27:1250-1256

14. Harrigan JA, Bohr VA (2003) Human diseases deficient in RecQ helicases. Biochimie 85:1185-1193

15. Hauben El, Arends J, Vandenbroucke JP et al (2003) Multiple primary malignancies in osteosarcoma patients. Incidence and predictive value of osteosarcoma subtype for cancer syndromes related with osteosarcoma. Eur J Hum Genet 11:611-618
16. Hopyan S, Gokgoz N, Poon R et al (2002) A mutant PTH/PTHrP type I receptor in enchondromatosis. Nat Genet 30:306-310

17. Ishikawa Y, Miller RW, Machinami R et al (2000) Atypical osteosarcomas in Werner syndrome (adult progeria). Cancer Sci 91:1345-1349

18. Ji J, Hemminki K (2006) Familial risk for histologyspecific bone cancers: an updated study in Sweden. Eur J Cancer 42:2343-2349

19. Li FP, Fraumeni JF, Mulvihill JJ et al (1988) A cancer family syndrome in twenty-four kindreds. Cancer Res 48:5358-5362

20. Lindor N (2002) Rothmund-Thomson syndrome In: Fletcher C, Unni K, Mertens F (eds) Tumours of Soft Tissue and Bone. IARC Press, Lyon, p 365

21. Maccarthy A, Bayne AM, Draper GJ et al (2009) Non-ocular tumours following retinoblastoma in Great Britain 1951 to 2004. Br J Ophthalmol 93:1159-1162

22. Mertens F, Unni KK (2002) Enchondromatosis: Ollier disease and Maffucci syndrome. In: Fletcher CDM, Unni KK, Mertens F (eds) WHO: Pathology and genetics of tumours of the soft tissue and bone. IARC, Lyon, p 356-357

23. Monnat RJJ (2002) Werner syndrome. In: Fletcher $C$, Unni K, Mertens F (eds) Tumours of soft tissue and bone. IARC, Lyon, p 366-367

24. Moppett J, Oakhill A, Duncan AW (2001) Second malignancies in children: the usual suspects? Eur J Radiol 38:235-248

25. Muftuoglu M, Oshima J, Von Kobbe C et al (2008) The clinical characteristics of Werner syndrome: molecular and biochemical diagnosis. Hum Genet 124:369-377

26. Olivier M, Goldgar DE, Sodha N et al (2003) Li-Fraumeni and related syndromes. Cancer Res 63:66436650

27. Pansuriya TC, Kroon HM, Bovée JVMG (2010) Enchondromatosis: insights on the different subtypes. Int J Clin Exp Pathol 3:557-569

28. Ranger A, Szymczak A (2009) Do intracranial neoplasms differ in Ollier disease and maffucci syndrome? An in-depth analysis of the literature. Neurosurgery 65:1106-1115

29. Rozeman LB, Sangiorgi L, Briaire-De Bruijn IH et al (2004) Enchondromatosis (Ollier disease, Maffucci syndrome) is not caused by the PTHR1 mutation p.R150C. Hum Mutat 24:466-473

30. Schwartz H, Zimmerman N, Simon M et al (1987) The malignant potential of enchondromatosis. J Bone Joint Surg Am 69:269-274

31. Silve C, Juppner H (2006) Ollier disease. Orphanet J Rare Dis 1:37

32. Tinat J, Bougeard G, Baert-Desurmont $S$ et al (2009) 2009 Version of the Chompret criteria for $\mathrm{Li}$ Fraumeni syndrome. J Clin Oncol 27:e108-e109

33. Tucker MA, D'angio GJ, Boice JD Jr et al (1987) Bone sarcomas linked to radiotherapy and chemotherapy in children. N Engl J Med 317:588-593

34. Wang L, Gannavarapu A, Kozinetz C et al (2003) Association between osteosarcoma and deleterious mutations in the RECQL4 gene in RothmundThomson syndrome. J Natl Cancer Inst 95:669-674

35. Wang LL, Levy ML, Lewis RA et al (2001) Clinical manifestations in a cohort of 41 Rothmund-Thomson syndrome patients. Am J Med Genet 102:1117

36. Werner O (1904) Über Katarakt in Verbindung mit Sklerodermie. Inauguraldissertation. Königliche Augenklinik der Königl. Christian-Albrechts-Universität Kiel 